\title{
Communicating Library Values, Mission, Vision, and Strategic Plans through Social Media \\ Doralyn Rossmann
}

\section{Abstract}

Social media communication needs to be aligned with your library's broader communication plan so that user experience is consistent with messaging across all library venues. Ideally, social media, along with all library communication points, include language from your library's values, mission, and vision statements and strategic plan. This article will outline two critical pieces to forming your social media communication strategy: Making a social media plan and applying social media optimization (SMO) to your library's Web pages. Once set up, your plan and SMO can easily become part of your library's routine when posting to social networks and creating and editing Web pages.

\section{Introduction}

In the first article in this series, I outlined an approach for libraries to craft a values statement, a mission statement, a vision statement, and a strategic plan which, ultimately, inform the creation of a communication plan. Cumulatively, I refer to the values, mission, and vision statements and your strategic and communication plan as your "S\&P." The language, ideas, and priorities identified in these statements and plans can become embedded in the library's organizational culture, including the library's approach to social media.

Communicating through social media networks has its own unique challenges because of platform-specific limitations such posting-length limits and organizational accounts being treated differently than individual's accounts, as well as platform-specific enhancements like embedding audio and visual media and being able to tag others in the posts.

Your library's social media communication with your communities can manifest itself in many ways. This communication may be through your library's social media posts, from information shared about the library by members of your community on social networks, or via interactions with your community members on those networks. Other types of communication include how your library's content is displayed to the user in social networks based on the way the content has been optimized for such networks. While many libraries have some sort of social media presence, it can easily be isolated or uncoordinated with other communication efforts. Much more than a marketing tool, social media can be used to build community and tell your library's story in keeping with all of your other points of communication. Social media communication needs to be aligned with your library's broader communication plan so that user experience is consistent with messaging from other library venues which also use language from your S\&P.

This article will outline two critical pieces to forming your social media communication strategy: Making a social media plan and applying social media optimization (SMO) to your library's Web pages. Once set up, your plan and SMO can easily become part of your library's routine when posting to social networks and creating and editing Web pages. 
Before engaging in social media efforts, consider the current social media landscape and the library's participation in it. Given the recent revelations about the manipulability of social media networks through automated accounts (a.k.a. bots) and through the algorithms used by the network developers themselves, it is important to consider the role of the library in these networks, whether participation in these networks aligns with the library's values, and the reasons why the library would be active in these networks.

\section{Make a Social Media Plan}

Objective: Create a consistent approach to the library's participation on social media networks in alignment with the library's S\&P

To ensure you have an intentional communication approach to your social media efforts, a social media plan can help keep your social media voice consistent, authentic, and can make it easier for anyone on your staff to share responsibility for the library's social media efforts. It should be regularly updated and revised as staffing changes, as your library adjusts which social networks it is active on, as features change, and, of course, to reflect the latest language used in your S\&P.

Your social media plan can start with some overarching social media goals and defined communities to keep you focused on what you intend to do and not do on those networks and who you intend to reach. Using language pulled directly from the values statement for Montana State University, the following approaches to your social media content, tone, and tenor might be established: ${ }^{1}$

- Accountability: Expand beyond the concept of "Library as Building" by participating in social networks, emphasize "Library as Services" and "Library as Resources" to students, researchers, faculty, and to the greater library, university, and Montana community.

- Diversity, equity, and inclusion: Seek out and engage individuals and groups through social media who may be underrepresented or underserved by the University to give a voice to their needs and to respond with desired services and resources.

- Empathy: Promote feedback about existing and potential services and resources that allows the community to shape the library's offerings. Develop relationships through social media that will ultimately allow existing university communities as well as newlybuilt online communities to discover a library that is more relevant, approachable, valuable, and vital, with resulting increases in overall awareness and use of library services and library resources.

- Inquiry and innovation: Create an environment through social media networks that encourages a collaborative and enthusiastic approach to the pursuit of knowledge. Leverage new social media networks to forge cross-disciplinary and cross-cultural collaborations to create new ideas and ways of knowing.

- Openness and access: Engage a growing and active university community by expressing the services, collections, and personality of the library and the library employees through social networks.

- Teaching and learning: Provide training and advocacy for library and campus teaching resources and services through social networks.

- Transparency and communication: Serve as social media leaders on campus and in the community by developing expertise through research, real-time interactions, platform and trend awareness, instructional and teaching sessions, and successful online community building. 
Then, you can create a section for each social network your library uses with your approaches to using and engaging each network clearly outlined. Such a plan could include:

- Name of the social media network

- Person(s) responsible for posting

- Community Focus

- Goals

- Values

- Activity Foci

- Tone and Tenor

- Posting Frequency

- Posting Categories

- Services and Resources

- Ongoing Series

- Handle/Account name and location of password

A completed version of this plan could might look like:

- Name of the social media network: Twitter

- Person(s) responsible for posting: Ed and Abi

- Community Focus: Undergraduates at MSU and residents of Montana

- Goals: To connect with undergraduate students about how the library can contribute to their student life, to listen to students about how their library might serve them, to expose our community to our digital and special collections which have a focus on Montana so they use these resources more.

- Values: People; User-centeredness; Openness and access; Teaching and learning

- Activity Foci: Present a welcoming and responsive physical and digital library environment; Provide convenient access to extensive collections; Provide positive learning outcomes and develop users information literacy abilities.

- Tone and Tenor: Empathy; Transparency; People

- Posting Frequency: Daily, including original posts, shares of stories, retweets, replies, likes

- Posting Categories: Highlight information resources representing diverse communities; Promote e-databases and how they can be used for specific courses or majors; Show spaces or resources that have been optimized for accessibility; Show different kinds of spaces in the library; Share materials from digital collections, especially those which have been optimized for social media sharing through search engine optimization; Cross-tagging MSU Library partners

- Services and Resources: Partnerships with TRAILS and other MSU departments; Spaces/Service locations in library building; Collections; Research, Publication, and Data Services

- Ongoing Series: Where are we in the library?; Digital collections in support of specific days/events (e.g. Native American Photo Collection shared on Indigenous Peoples Day); \#WaybackWednesday \& \#ThrowbackThursday

- Handle/Account name and location of password: @msulibrary, password on social media group's intranet space 
In this example, we took a look at the S\&P and filled in each of the categories. ${ }^{2,}{ }^{3}$ The Goals section aligns with the MSU Library Vision Statement. The Values and Tone and Tenor sections were drawn from the MSU Library Values Statement. The Activity Focus items are objectives from the MSU Library Strategic Plan and the Posting Categories and the Services and Resources are initiatives in support of objectives from the Strategic Plan. The remaining categories depend on your library's time and resource capacity for participating in social media networks and can be tweaked in the future based on experiences with that specific social media platform. You can walk through this same process for each of your social media platforms to develop a comprehensive social media plan.

Going through this exercise of embedding elements of library's S\&P in your social media plan results in making your S\&P an active part of the language and content used by the library in social media networks. One other consideration that may align with your values is the role of the library in educating its community about the challenges of social media networks including privacy, algorithms used by the networks to control what information is displayed to you, and the potential of bots or fake accounts to be present in these networks. Ethically, when libraries participate in these networks, there is an implicit endorsement of the use of these networks. One of MSU Library's values is "Transparency and Communication" which suggests that it should be transparent about how it uses any data from these networks, that it should take opportunities to educate users in these networks, and it could consider encouraging the community to use alternative-to-mainstream networks such as Mastadon. ${ }^{4}$ Also, consider posting your social media plan to your library's Web site so that you can be transparent in your efforts. Whoever manages the library's social media efforts will likely be asked to post things for the library to social media from time to time which may be outside the scope of the social media plan. Use your social media plan to keep your posts within the elements of your S\&P and remind anyone asking you to post that it needs to adhere to the social media plan.

\section{Implement Social Media Optimization}

Objective: Create a consistent approach to the presentation of library content in social media networks in alignment with the library's S\&P

The other part of the social media sphere which is part of your communication plan adheres to the values of social media optimization (SMO). Rossmann and Young (2016) provide a comprehensive step-by-step guide for implementing social media optimization for your library. Here, we will explore some of those same concepts with the very intentional focus on carrying out your library's S\&P as part of your SMO.

\section{Background of SMO}

As Rossmann and Young describe, "The primary goal of SMO for libraries is to encourage social media engagement and content sharing through the major social networks that constitute our users' learning and research environments. The primary outcome of SMO is an engaged community of library users. An engaged community can then generate many secondary effects, including increased Web traffic to library pages and increased library resources usage, as well as establishing and reinforcing the library as a trusted member of the community."

There are five principles of SMO which include approaches to sharing content on social media networks and ways to optimize your library's Web content for sharing on these networks. Thus, an effective communication plan includes what you choose to communicate on social 
networks, what you optimize for others to share on these networks, and how you optimize your information for interpretation by the algorithms used in these networks.

I recommend creating an SMO strategy that will accompany your strategic plan and uses language found throughout your S\&P. From your strategic plan, consider the three ways you might use SMO: (1) Library Initiative Self-Promotion: decide which initiatives themselves are well-suited for your library to talk about on social media networks (such as a your library posting on Facebook about the remodeling of group study space in the library), (2) Library Initiative Others-Promotion: identify which initiatives themselves are well-suited for other people to share on social media networks (such as library users Tweeting about a new self-checkout station in the library), and (3) Library Content More Shareable: select which initiatives could benefit from improving shareability on social networks (such as adding coding to your digital collections Web pages to optimize those collections for display when posted to Facebook or Twitter). Some of your initiatives may not fit easily into any of these categories which means you do not need to apply any social media optimization techniques to them.

There are five principles to SMO. Each of these principles is described in the following section, with techniques to incorporate parts of the language from your S\&P, as appropriate.

\section{Principle 1: Create Shareable Content}

The first principle of SMO is to Create Shareable Content. Creating Shareable content means making the information your library provides online optimized for sharing on social media networks - both for sharing by the library and for others to share. Creating shareable content may be new content about your strategic initiatives or it may be optimizing existing content for shareability. This principal can help you with all three ways of applying SMO identified at the beginning of this section.

Here is one objective taken from the MSU Library's strategic plan:

Objective: 2.1. Create useful, dynamic, and accessible digital and physical spaces

Measure name: Digital Space Accessibility

Frequency of activity: Annually

Description: WAVE \& Accessible social media (https://accessibility.umn.edu/tutorials/accessiblesocial-media)

Baseline for assessment: Accessibility audit

Target goal: Reduce number of WAVE errors, increase \# items with social media optimization \& and which are accessible

\section{Proposed coordinator: Andrea}

To help meet this objective to "create useful, dynamic, and accessible digital and physical spaces," it would be useful to learn about how digital library content posted to social media networks is rendered for screen readers for those with visual impairments. Facebook and Twitter allow you modify your Web pages for optimal sharing with coding called Facebook Open Graph Tags and Twitter Cards. The developer site for Twitter cards walks users through each of the steps for implementing the cards. ${ }^{6}$ One of the tags that can be added to images is an "alt" tag for an image shared on Twitter. ${ }^{7}$ With this information added, any Tweets using the Web 
address, or URL, for that image will include the title you put in the alt tag which can be interpreted by screen readers, making for more of your library content which is shareable and accessible.

You will want to create social media content specifically focused on the objectives for your strategic plan and which uses language from the parts of your S\&P. It can be helpful to take your strategic plan and add columns to it to indicate if it will be shared on social media networks, which networks, and what the content might include.

\section{Principle 2: Make Sharing Easy}

The next principle of SMO is to Make Sharing Easy. The idea behind this principle is to ensure the content you create has low barriers for others to share, re-share, or find through searching. For example, hashtags can be used on platforms like Twitter and Instagram to make content findable by anyone who follows or searches for that hashtag. If you're promoting the library's efforts to be open and transparent, take a look at the social media network where you will be posting and search possible hashtags and how they are being used. The hashtag "\#transparency" seems to be dominated by exercise and fitness while "\#governmenttransparency" seems more aligned with what a publicly supported university might have in mind. Also consider using multiple hashtags to make your content more discoverable and to send messages to your community of the sentiment of your posts.

Adding Facebook Open Graph Tags and Twitter Cards to your Web pages is another way to make your content more easily shared. Facebook Open Graph allows you to control how any URLs shared from you library's content is displayed on Facebook, including any images, and descriptions which, otherwise, are up to Facebook's algorithms to decide how information from a URL is interpreted. ${ }^{8}$ Likewise, Twitter Cards provide the opportunity to surface pictures, video, and media to your Tweets along with a brief description of the content and your Library's Twitter handle as the owner of that content. Adding Twitter Cards to your Library's content also allows you to get analytics about engagement with Tweets other share about your content as well as your own sharing.

Take a look at your strategic plan and consider where the addition of Facebook Open Graph Tags and Twitter Cards might be most beneficial for your own sharing of library content as well as what others share. Also consider what elements of your strategic plan might benefit from targeted social media campaigns, including the use of hashtags to surface content.

\section{Principle 3: Reward Engagement}

The third SMO principle is to Reward Engagement. This principle requires effort on your Library's part to watch for library mentions, shares, hashtags, check-ins, etc. be aware of either mentions of the library or to learn about user needs which could be fulfilled by the library.

For example, the library might be mentioned in photograph on Instagram through location tagging within the post. If the user makes their post public or if the user is followed by the library, then the library would be able to find such a post by searching the library as a location on Instagram. Depending on the tone of the post, the library could choose to reply to the person posting as deemed appropriate. Hopefully, any such responses to posts offer the opportunity to espouse the values found in the Library's values statement such as empathy, transparency and openness, equity, and diversity, Using these exact value terms in your replies, whether direct responses or reposts, can keep your library's voice and messaging consistent and in alignment with your broader conveyance of library values. In addition to monitoring for 
direct mentions of the library or the library as a geotagged location, some other examples of rewarding engagement include: responding to user needs (such as a user posting to their community that they don't know how to find articles for a class assignment), commenting on something the user posted (such as something good they did for someone else), following users, and following back users when they follow the library.

It is worth adding a caveat with the principle of Rewarding Engagement. This principle suggests directly engaging with your community. But, what if members of the community do not expect such engagement from the library or do not want such engagement? For example, maybe a user posts a Tweet on Twitter and the library retweets the posting. Maybe that user did not want that Tweet shared more broadly or shared by the library. While social networks have a lot of opportunity for social sharing, issues of privacy should still be of concern. It can be useful to consider the context of posts, the intended audience of the posts, and the reason for the library to engage with the post.

\section{Principle 4: Proactively Share}

Proactively Sharing is the fourth principle of social media optimization. The library can proactively share in a variety of ways. First, by following the posting categories and schedule outlined in the social media plan created in the last section, the library has a plan in place to surface content that espouses the library's values and strategic plan progress. Adding geotags to posts can help surface content to others who monitor locations or to others who check-in to those locations. The library does not need to restrict itself to sharing content from the library. Keeping in mind the parts of the S\&P, consider what external content might be logical for the library to share. For example, if providing your community with easy access to high-quality information resources is a priority, it might help to surface links to open educational resources which you have deemed to be accurate and reliable.

\section{Principle 5: Measure Use and Encourage Reuse}

The final principle of social media optimization is to Measure Use and Encourage Reuse. The first part of this concept is to look at the data you have available to see what is getting engagement with members of your community. Looking at your S\&P, you might have a goal to increase access to and use of your library's digital collections. To support this effort, you could look at Google Analytics to review your Web site traffic and the source of that traffic such as the referrer (search engines, social media) or geographic region of the world. ${ }^{9}$ Twitter Analytics tells you about your Twitter account engagement, and, for any content on your Web site which has Twitter Cards enabled, they will be a part of the analytics. This information can help you discover when others have shared your library's Web content via Twitter. ${ }^{10}$ This information offers the opportunity to follow the earlier reviewed principle of Reward Engagement by possibly interacting with the account that shared the library's content by liking, retweeting, or commenting on the post.

The other part of this principle includes the idea of Encouraging Reuse. One really easy way to encourage reuse is to have clear language on your library's Web site that explains how materials can be used. For example, if you have digital images from your library's special collections posted on your Web site, you could add a Creative Commons license to encourage people to reuse the images and to give attribution to the library in using the images. ${ }^{11}$ Looking at the MSU Library, this would espouse the values of openness and transparency and such language could be used in the statement about the Creative Commons licensing. 


\section{Further Discussion}

The topics discussed here emphasize the importance of having a social media communication plan which is informed by the statements and plans created by your organization. This article builds on the first in the series which outlined the development of your S\&P and how those inform the general communication plan. The next article in this brief series will cover the details of forming a budget communication plan which aligns with your overall communication plan. All of these plans go beyond traditional communications and incorporate your S\&P language into other areas of communication which reflect the work and values of the library. If there are additional areas you would like me to cover in future articles, please contact me at email (doralyn@montana.edu) or twitter (@doralyn). Happy communicating!

\section{Bibliography}

Creative Commons. "About the Licenses." 2019. https://creativecommons.org/licenses/.

Facebook. "A Guide for Sharing for Webmasters." 2019.

$$
\text { https://developers.facebook.com/docs/sharing/webmasters/. }
$$

Google. "Analytics." 2019. https://analytics.google.com/analytics/web.

Montana State University Library. "Mission, Vision, and Values.” January 31, 2018.

http://www.lib.montana.edu/about/mission-vision/index.html.

Montana State University Library. "Strategic Plan: Our Vision for the Future." January 31, 2018. http://www.lib.montana.edu/about/strategic-plan/.

Rossmann, Doralyn. “Creating an Organizationally Embedded Strategic Communication Plan for Libraries." Library Leadership \& Management 33, no. 2 (February 1, 2019). https://journals.tdl.org/llm/index.php/llm/index.

Rossmann, Doralyn, and Scott W.H. Young. Social Media Optimization: Principles for Building and Engaging Community. American Library Association, 2016.

https://journals.ala.org/index.php/ltr/issue/viewlssue/617/379.

Twitter. "Alt Text Support for Twitter Cards and the REST API." March 29, 2016.

https://blog.twitter.com/developer/en us/a/2016/alt-text-support-for-twitter-cards-andthe-rest-api.html.

Twitter. "Analytics." 2019. https://analytics.twitter.com/.

Twitter. "Optimize Tweets with Cards." 2019.

https://developer.twitter.com/en/docs/tweets/optimize-with-cards/guides/getting-started. 
Doralyn Rossmann (doralyn@montana.edu) is Head of Digital Library Initiatives at Montana State University

Published: June 2019

References

${ }^{1}$ Montana State University Library. "Mission, Vision, and Values." January 31, 2018.

http://www.lib.montana.edu/about/mission-vision/index.html.

2 Ibid.

${ }^{3}$ Montana State University Library. "Strategic Plan: Our Vision for the Future." January 31, 2018.

http://www.lib.montana.edu/about/strategic-plan/.

${ }^{4}$ See https://mastodon.social/about.

${ }^{5}$ Rossmann, Doralyn, and Scott W.H. Young. Social Media Optimization: Principles for Building and Engaging Community. American Library Association, 2016.

https://journals.ala.org/index.php/ltr/issue/viewlssue/617/379.

${ }^{6}$ Twitter. "Optimize Tweets with Cards." 2019. https://developer.twitter.com/en/docs/tweets/optimize-withcards/guides/getting-started.

${ }^{7}$ See Twitter. "Alt Text Support for Twitter Cards and the REST API." March 29, 2016.

https://blog.twitter.com/developer/en us/a/2016/alt-text-support-for-twitter-cards-and-the-rest-api.html.

${ }^{8}$ Facebook. "A Guide for Sharing for Webmasters." 2019.

https://developers.facebook.com/docs/sharing/webmasters/.

${ }^{9}$ Google. "Analytics." 2019. https://analytics.google.com/analytics/web.

10 Twitter. "Analytics." 2019. https://analytics.twitter.com/.

${ }^{11}$ Creative Commons. "About the Licenses." 2019. https://creativecommons.org/licenses/. 